\title{
Unobtrusive Student Collaboration during Lectures with Smartphones
}

\author{
Mark Reilly \\ Flinders University \\ Bedford Park, SA \\ Australia \\ m.reilly@flinders.edu.au \\ Haifeng Shen \\ Flinders University \\ Bedford Park, SA \\ Australia \\ haifeng.shen@flinders.edu.au
}

\begin{abstract}
The lecture is still the primary teaching and learning form in university and will remain so due to its cost-effectiveness. However, student disengagement caused by the traditional didactic style of lecture - with its one-way information flow allowing little or no interaction prevents some students from taking full advantage of this learning paradigm. Some work has been done to address this issue by re-designing lectures to incorporate more lecturerand-student interaction, but has yet to be widely adopted. In this paper, we present an approach to applying a student-centred collaborative learning pedagogy into the lecture environment through a novel real-time collaborative note-taking application GroupNotes which encourages students to proactively engage themselves by means of student-andstudent interaction on smartphones. Benefits of this small group interaction is reducing the feeling of isolation that students feel in large lecture theatres through a sense of belonging as well as the incentive to remain on task through peer pressure from within that group.
\end{abstract}

Smartphone, collaboration, education

\section{INTRODUCTION}

We present our progress towards a technology assisted solution to furthering undergraduate university student's engagement in lectures. Our solution makes use of smartphones due to their expected forthcoming dominance of the mobile phone market as well as the relative lack of cost, both monetary and time, to the university and the students. The available storage, power, connectivity options, screen size and speed of text entry have progressed to the point where we feel they can make a valuable contribution in those lectures where, for many different reasons, students do not engage fully, for the entire session, or in some cases, even turn up.

A lecture is still the primary teaching and learning paradigm in most universities, and is likely to remain so in many years to come. The most important benefit of the lecture to the university is its cost-effectiveness as a model of delivery to an audience ranging from tens to hundreds, and perhaps even thousands. It is also where the uniform delivery of information, not found in the standard texts, and also the possibility to motivate and inspire students exist [1].
However, to many the lecture is also seen as a sub-optimal learning environment with issues ranging from the shape of the venue promoting the audience as spectators rather than participants through to the sense of loneliness and isolation many students report from being a lone voiceless participant in a room of many, and up to hundreds.

Technical solutions to increase student engagement have been tested before, some are still in place however they all have a cost involved, either to the university to ensure learning material is developed to make use of the technology, or to purchase or subsidise sufficient quantities of the devices to ensure that an education divide does not occur between those willing and able to afford the new devices. The costs are not always exclusive to the university, in many cases there is also a cost to the students to firstly purchase, hire or rent the device and then further charges to actually use the devices, such as SMS charges or internet access fees. A further opportunity cost, if the device is not seen to be useful by the student is the effort required to understand and effectively use the device beyond the bare minimum requirements instead of doing something else more desirable.

Our primary objective is to encourage students to proactively engage themselves in a lecture by 
encouraging them to actively take notes during the session, an effective learning technique that aids memory of the lecture by fostering encoding, articulation and rehearsal [1].

Our proposal is to allow a small group of students to participate in a real-time collaborative notetaking session using their own smartphones, and motivate, assist, and monitor each other in order to actively learn and keep themselves engaged during the lecture. In this paper, we present a technical approach that allows a small group of students to collaboratively take notes of lecture content on slides, review and rectify each other's notes, comment on the lecture or notes, and ask or answer questions within the group all in real time during a lecture, with nothing more than their everyday communication device - the smartphone, and do so in a way that does not disrupt the lecture, either for the lecturer, or for other students. We are in the process of designing and implementing this application, initially using an Android-based smartphone, by adopting the usercentred design methodology in order to maximise user acceptance and minimise the learning curve. Human factor study including user interface, evaluation and usability study is ongoing, while pedagogical study including workload, cognitive load, engagement level, learning outcome, and usage scenarios will follow.

The rest of the paper is organised as follows.

Section 2 describes the pedagogical background and the proposed approach, in turn followed by the motivation and design informing the application user interface. We then present the System Architecture followed by the innovative collaborative note-taking. Finally we conclude the paper with a summary of major contributions and future work.

\section{PEDAGOGICAL BACKGROUND AND PROPOSED APPROACH}

We proposed that a smartphone-based approach to increasing student engagement in lectures is a viable and, most importantly, an accessible option for today's students Reilly and Shen (2011). A smartphone-based collaborative note-taking application will provide a platform for small groups to create a self-motivating, social learning environment allowing them to collaborate silently in lectures while providing the necessary options and customisation to cater for the different learning styles of the individuals within those groups.

The choice of the smartphone as the platform for a collaborative tool suitable for use within a lecture environment is due to the predicted ubiquity of such devices. At present the majority of university students either own or have access to a mobile phone [2] and IDC [3] predicted that by the start of 2010 sales of smartphones within Australia would exceed $50 \%$ of new sales. While larger devices such as a tablet PC may provide a more usable learning environment [4], [5], not all students are able, or willing to purchase the device, bring it to university with them, and then actually use it in the lecture environment.

The smartphone is attractive to the student as there is no imposition associated with purchasing or carrying an additional device, or in learning to use it or the new software since they have already learnt how to use the device voluntarily. There will be no additional monetary costs to use the device in the lecture environment as wireless access is already provided free therefore removing a major concern of students [2].

Staff-student contact time at university is dominated by the lecture because it is recognised for its ability to uniformly deliver information not found in the standard texts, as well as providing the opportunity to inspire and motivate students ranging from hundreds to perhaps thousands [1]. Yet many students view some or all of them as a waste of time [6] and either cease attending those lectures, or lose interest in as little as 20 minutes [7]; [8] of a lecture due to no interaction with either the lecturer or other students in the vicinity [1]. If the lecturer does not take special measures to engage students by, for instance, asking or answering questions, quick breakout sessions, quizzes, or simply telling a joke, their attention may wander.

The actual lecture environment itself is sub-optimal as it promotes the feeling that the students are at a performance. The shape of many lecture halls is that of an amphitheatre where the lecturer is far removed from the audience of students who are expected to sit in silence while the lecturing "performance" takes place rather than an atmosphere which promotes participation. Students are disadvantaged in several ways with this environment, most notably because with the implied or actual prohibition on speaking, there is no opportunity to immediately interact with their fellow students, for clarification or validation of their understanding on the lecture content. Students feel isolated, part of an "anonymous mass" [9]. This change of teaching paradigm away from social learning as a member of an interactive (high school) classroom to that of a (mostly) passive audience member is seen as a cause of students failing to successfully complete their studies. Note-taking during lectures is established as an important part of the process of codifying lecture content into knowledge that can be retrieved later. 
The current technology, in the form of soft keyboards such as Swype [10], Swiftkey [11] and T9 Trace [12] allow text entry speed comparable or even faster than pen-and-paper handwriting. Our proposal is based on this observation [13] and incorporates opportunities for the social learning attributes of challenging or validating one's current "knowledge" against others in a group in real time, which motivates a student to remain on task and to produce quality work. The application will also provide for the individuals who prefer to work alone, or who cannot keep up with multiple students during the lecture but would like to be part of a group with sharing of knowledge. In this instance, the student works alone, but stays motivated to produce quality work and to remain on task by the prospect of receiving multiple sets of notes from their other group members at the end of the lecture. When the students are fully engaged, their attention span seems infinite, regardless of the teaching method [14]. In this paper, we aim to design and implement such a smartphone application GroupNotes, a collaborative note-taking application that allows students to teach, correct, motivate and learn from their peers during the lectures.

In a large number of lectures the lecturer actively pushes information to a mass group of students, who passively process the information alone in an attempt to build the relevant knowledge for themselves. The lack of feedback or interaction, at the time when it is most needed for a student to process relevant information during the lecture is a major factor toward student disengagement, which consequently causes negative impacts on their willingness to attend lectures and on their learning outcome. If a student cannot ask the lecturer [6] or their peers critical questions at the right time, the teaching moment, to reconcile their own understanding they may lose interest and find other ways to occupy themselves, such as doodling, sleeping, texting, twittering etc., or just getting up and leaving [15].

Taking into account that approximately $50 \%$ of contact time between students and teaching staff is comprised of lectures, there is a real possibility that as much as $30 \%$ of all staff - student contact time is essentially wasted.

In recent years, much attention has been paid to re-designing lectures in a bid to incorporate lecturer and student interaction (vertical interaction) by taking advantage of new teaching gadgets such as clickers or mobile phones [16]; [4]. Empirical evidence as to how student engagement can be improved by this approach as well as to their effectiveness in increasing student learning outcomes is yet to be proven [17] conclusively. For this reason many lecturers have yet to be convinced of making extra effort to re-design their lectures in order to harness these new technologies. Our approach is complementary to the current endeavour, but from an orthogonal perspective, i.e., student-and-student interaction, (which we call horizontal interaction). This has been practiced in small-class tutorials, workshops, or practical sessions, but virtually prohibited in large-class lectures because it does not fit well into the lecture context and is likely to disrupt other students in the vicinity of those students involved in the interaction. To make this approach viable in lectures, we specifically want to address the following issues.

First, this approach is based on a student-centred collaborative learning pedagogy, which is proven effective in group-based learning tasks or activities in small classes [4]; [9]. However, application of student-student collaborative learning to large-class lectures is still under exploration and we want to study its positive effect on improving student engagement and learning outcomes and at the same time investigate any potential negative effects that may develop.

Second, the approach is also based on an active learning pedagogy, where students take a proactive role in engaging themselves rather than being passively kept engaged by the lecturer. Notetaking is an effective learning technique particularly in lectures, where students engage themselves to make sense of the lecture by documenting their understanding or misunderstanding of the information passed on by the lecturer in their initial knowledge building process. However, as notetaking is largely a personal choice rather than a curriculum requirement, students who do not take notes during a lecture may not be actively engaged unless other means are provided. We want to investigate how to motivate students to proactively take notes in a more engaging and stimulating way.

Third, we want to address the issues responsible for the slow uptake of hardware-based teaching innovations in lectures by investigating smartphone-based horizontal interaction. This approach does not require any change from either the lecturer or the student if they do not want to; that is, it does not require the lecturer to re-design the lecture and it adds no extra burden to students, either cost to own, time to learn, or effort to bring with them, as an increasing percentage of the student population have already upgraded, or are considering upgrading to smartphones which are always with them [3].

In a nutshell, Seven Principles for Good Practice in Undergraduate Education [18] advocates developing reciprocity and cooperation among students, using active learning techniques, 
emphasising time on task, and giving prompt feedback. While this ideal is from the teaching staff to the students, we aim to provide similar practices from each student to their peers in a collaborative learning group based on the hypothesis that the fun factor (e.g., smartphone against paper-and-pen, collaborative learning against individual learning) and the peer motivation (e.g., the desire to help peers or not to let peers down) will be driving forces towards students proactively engaging themselves in lectures. We need to systematically develop this technical approach and then test this hypothesis. For collaboration to be considered effective, the process must include discussion and reflection [19] which in turn leads to deeper and more meaningful learning.

We suggest the availability of collaboration on the smartphone platform will promote the ability of the student to understand, analyse and evaluate lecture content, identified as three of the Six Cognitive Learning Outcomes for Undergraduate Education [20], and therefore will help students achieve deeper understanding, leading to improved learning outcomes.

\section{USER INTERFACE MOTIVATION \& DESIGN}

Saville-Smith suggested that handheld computers can assist students' learning in a number of ways, including motivation and supporting both independent and collaborative learning [21]. Milrad identified useful features that mobile technologies provide for education in terms of social interactivity, individuality, connectivity and portability [22]. However, there are some identified obstacles for handheld computers and mobile devices to be effectively used in lectures, for example, the difficulty in bringing them along to lectures, the overhead in setting them up, the expense in owning them, and the perceived benefits they can bring about. For example, clickers are not yet proven [17], mobile phones were seen to be too limiting, PDAs were too expensive for their limited functionality [23], and tablets and laptops were seen to be too expensive, difficult to operate (due to limited space in the lecture environment), and a distraction to other users in the room. Generally speaking, students were unwilling or unable to own the specific devices, bring them to lectures or invest in learning how to use the devices or applications such as Livenotes [4] and NoteBlogger [5].

The current power and sophistication of handheld computers, or converged devices - the smartphones, such as the Apple ${ }^{\circledR}$ iPhone $^{\mathrm{TM}}$, Samsung ${ }^{\circledR}$ Galaxy S or HTC $\AA$ Desire, - are very attractive to the modern generation who expect instant information to be available to them whenever and wherever they are. Research on using smartphones for teaching and learning is emerging in recent years primarily due to increased self-ownership and the familiarity with their own devices. Combined with their technical sophistication (e.g. computation power, screen size, storage, friendly and easy-to-use interface etc.) and portability they remove the major obstacles to providing students viable technical solutions to their learning needs as evidenced with the recent introduction of Hotseat at Purdue University [24] and $M L I$ at the University of Oulu [25].

We want to harness the unique characteristics of smartphones to address the student disengagement problem in lectures. Where this proposed solution differs from those already discussed is that it aims to tackle the issue from the perspective of students being kept engaged by their peers in a small virtual collaborative learning group (i.e., group members may not necessarily be physically seated together) within a large lecture audience. In contrast, the majority of existing work has focused on students being kept engaged by the lecturer through increased two-way vertical interaction.

The cornerstone of the proposed solution is an application called GroupNotes, which will operate on smartphones, providing for a small group of students to interact with each other through taking, reviewing, commentating, and questioning notes for lecture content as it is being delivered. The application is based on the observation that the speed of text entry available on a smartphone through such innovations as on-screen soft keyboards like Swype and Swiftkey, predictive text, predictive next word, auto complete, auto correct and the ability to copy and paste means that an experienced user can now 'write' even faster on a smartphone than hand write with pen and paper, which alleviates the issues outlined by Ward [26] regarding the time involved in replicating the usual pen-and-paper notation methods onto electronic devices. In addition, the screen real estate of a smartphone, typically ranging from 3.5 to 4.3 inch at the moment, we think is acceptable for a few users to be adding content to the display surface simultaneously if the application's user interface is properly designed. The application allows real-time collaboration within a small group of students to validate and explore the meaning of lecture content, when it is being delivered, yet in a silent manner that does not disrupt either the lecturer or other students.

The user interface design of the GroupNotes application is concerned about issues such as utilisation of screen space, simplicity and ease-touse, efficient use of major functions, 
unobtrusiveness of minor functions, user control and freedom, and awareness of group work. We adopted the user-centred design methodology in order to maximise user acceptance and minimise learning curve. To that end, we did a needs finding survey among students enrolled in the Mobile Applications topic (a fourth-year computer science topic) and received 17 responses to our survey. These results that helped our user interface design of the GroupNotes application are shown in Figures 1-6. User interface evaluation and usability study are currently ongoing.

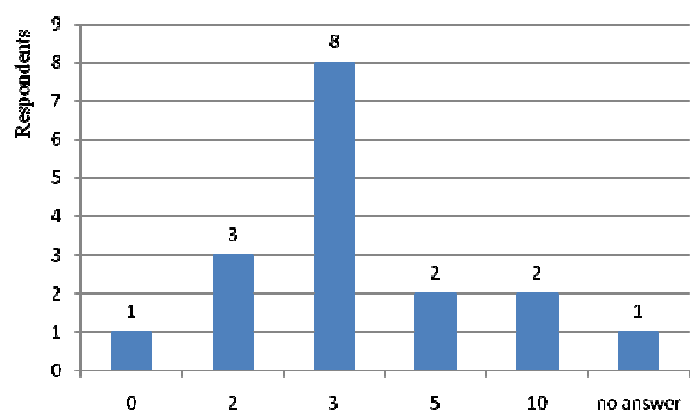

Fig. 1: Maximum group size (excluding oneself) in terms of cognitive load to keep up with others

First, a group consists of maximally 4 students and the screen is equally divided into up to 4 notes areas, one for each student. This design is based on a typical smartphone screen size of approximately 4 inches and our survey about the cognitive load involved in keeping up with the lecturer and other students in a group (Fig.1). The actual amount of screen space given to each student is dynamically adjusted according to the number of active students taking notes for a particular lecture slide as well as how much space each individual is requiring until all students are contributing equally and then the screen space is divided equally. When one's notes area is filled up, it can be scrolled up and down. Each individual is able to reallocate the amount of space on their own screen that is given to others in their group as required by flipping one's notes area to maximise, minimise, or normalise it, providing adequate flexibility in allowing students to use the application in a way that best suits them.

Second, while each member in a group usually works in their own notes area, it is possible that multiple members write to the same notes area, e.g., rectifying errors in a note or making comments on a note. Therefore, it is essential to clearly distinguish their contributions in the same notes area. In the GroupNotes application, each group member is associated with a unique text colour for self- identification as the survey (fig. 2) indicated that a majority of students prefer using colours to differentiate group members in terms of the screen space consumption and the clarity of user identification.

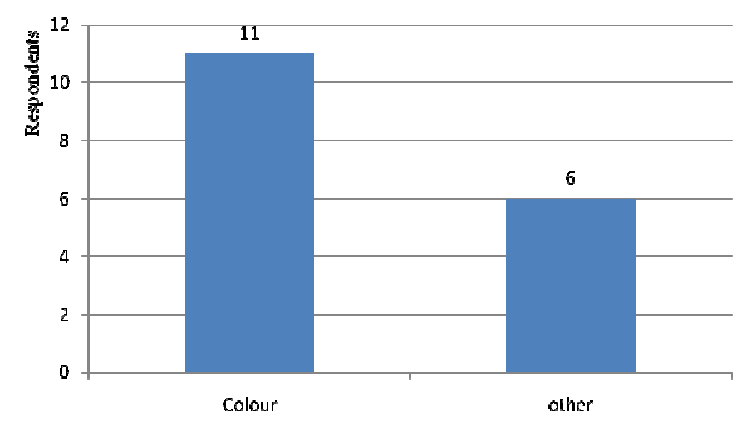

Fig. 2: User identification within a group

Third, notes are associated with each slide, but are not taken on the slide directly, mainly because: 1) the slide will fill up the entire screen space, making note-taking extremely difficult, 2) the current slide is projected on the wall during the lecture, 3) notes will be reconciled into the slide automatically at the end of the lecture by the post-processing service (to be elaborated on in the next section), and 4) a copy of the entire lecture slides can be downloaded to one's smartphone and viewed by a separate application in the rare case where one needs to refer to a slide other than the current one. The design is supported by the survey results shown in Figures 3-5. It is worth clarifying that because we did not explain the first point in the survey, the result shown in Fig. 3 is not very conclusive; but after we elaborated it in a subsequent session, 5 out of 8 students changed their mind in supporting separate notes.

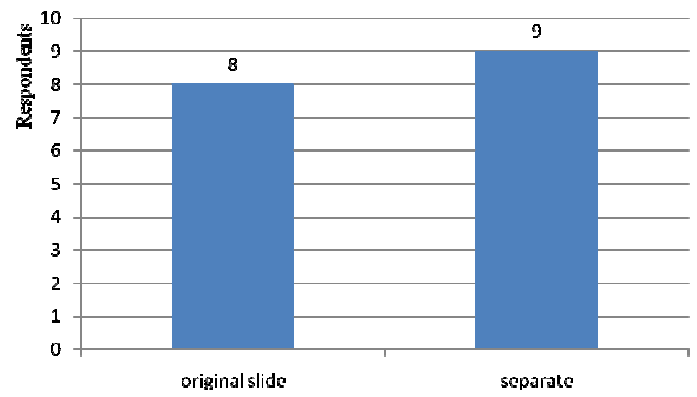

Fig. 3: Where should notes be taken? 


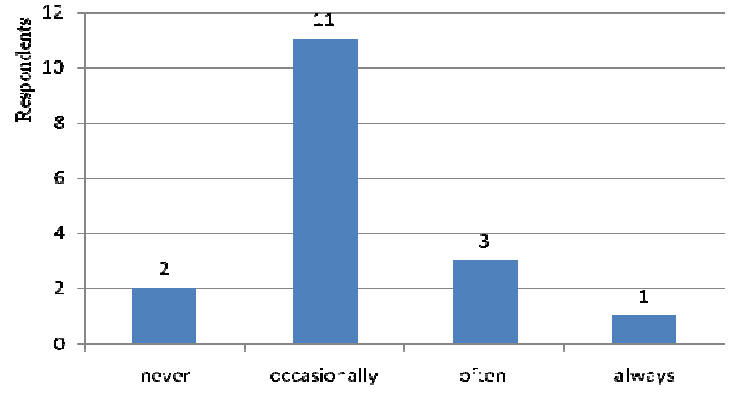

Fig. 4: How often is a different slide (not the one being projected on the wall) referred to?

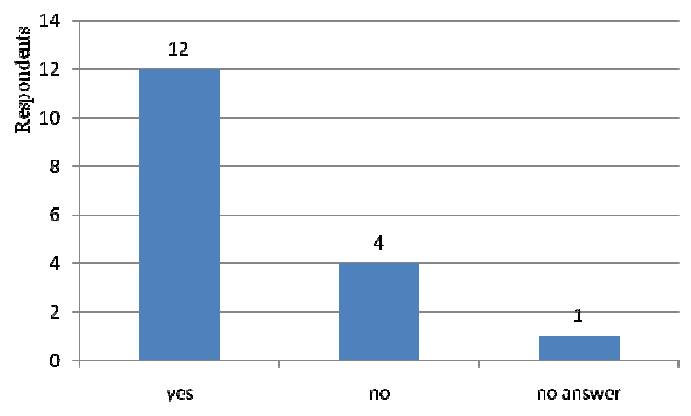

Fig. 5: Should copies of slides be available on the smartphone for reference during a lecture?

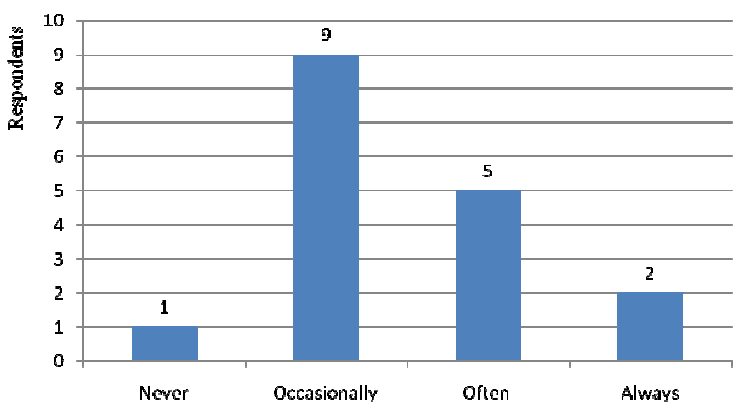

Fig. 6: How often is diagram-drawing needed when taking notes during a lecture?

Last, as suggested in the survey, diagram-drawing is only occasionally needed in a note-taking session (Fig.6).

Therefore, access to the drawing facility is supported in an unobtrusive way that: 1) the drawing toolbar is only made visible by tapping or flipping a notes area and hides automatically after the drawing task is done, and 2) diagrams are not inserted into a notes area as they would fill up the area, making note-taking difficult; instead, they are kept separate, but the notes maintain links to them so that they can be inserted into the right places of the notes automatically at the end of the lecture by the post-processing service.

The user interface design should also allow for adequate user control and freedom, ranging from individual use to cooperative use and collaborative use, depending on how students wish to use their devices and the application at any given time.

Some students may only use GroupNotes on an individual basis, not as part of a group, purely for the benefit of getting the digital form of the notes. Although individual use is not the scenario we would expect students to take on, it does serve the purpose of engaging students in lectures and allowing students to be in control of their own preferred learning styles, also students have a digital form of their own notes that can be used for revision.

Cooperative use of GroupNotes is where a student, though being part of a group, independently take notes for the entire lecture slides because they cannot manage the cognitive load involved in keeping up with their peers' contributions while at the same time listening to the lecturer and taking their own notes, or only for a subset of the lecture slides (e.g., slides 1-5, odd slides etc.) to take on a fair share of the workload in the group. In this instance each member's notes form part of the community notes [27] available to the group at the end of the lecture.

Collaborative use of GroupNotes usually involves participants taking different roles in a real-time collaborative note-taking session in order to maximise each one's strength and cognitive power. For example, a group involving 4 students: Adam, Bruce, Carol and Deng, where Adam is designated as the note-taker, who takes notes for the entire lecture, Bruce is designated as the reviewer, who reviews and rectifies the notes, Carol is designated as the commentator, who comments on the lecture and the notes, e.g., identifying the possible exam questions from paying attention specifically to the emphasis - written, facial, voice or mannerisms of the lecturer, and the fourth member Deng is designated as the questioner, who develops questions, which if answered would encapsulate the knowledge provided during the lecture.

Collaborative use of the GroupNotes application allows a group of students to flexibly and maximally share the workload, leverage each one's strength and expertise, and take advantage of the power of collaborative learning. Even top-tier students can benefit from small-scale 'The Wisdom of Crowds' [28], where cognitive diversity provides multiple views on the current subject matter that can challenge or validate the current 'knowledge' of the individual.

\section{SYSTEM ARCHITECTURE}

Fig.7 depicts the GroupNotes system architecture consisting of a GroupNotes Server, GroupNotes 
Apps on smartphones, and a GroupNotes Console on the lecturer's computer.

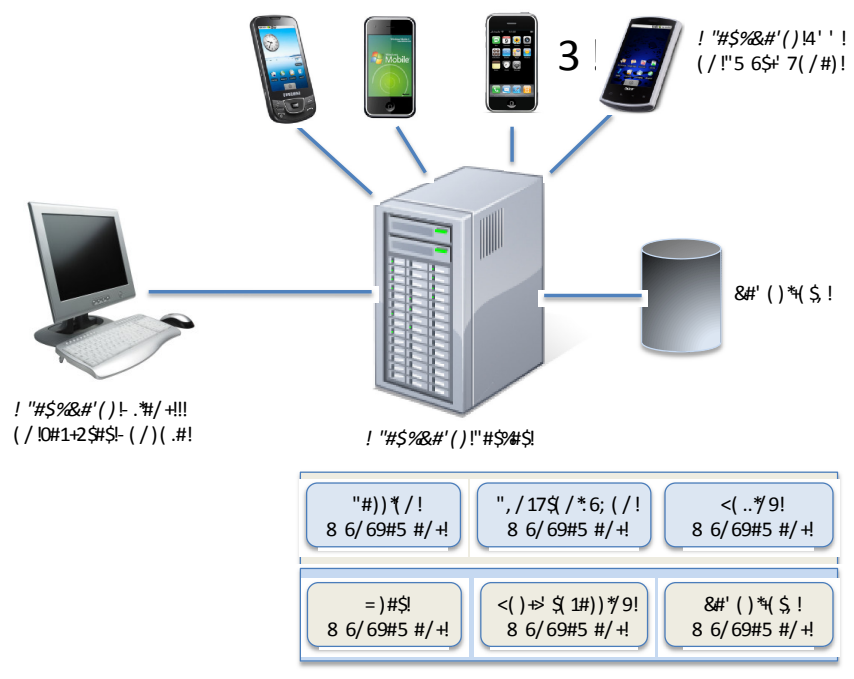

Fig. 7: The server provides a set of system services.

The services provided by the GroupNotes server can be divided into online and offline management. Online management consists of essential services required by the GroupNotes application and client during a lecture. In particular, session management is to create a collaborative note-taking session for each group and handle session-related activities such as joining/leaving an on-going session, saving notes of the session back to the repository, adjusting members' roles in the session, inviting someone outside the group to join the session, recovering from errors or application crashes and so on Synchronisation management handles everything related to shared access to a particular notes area, including optimistic concurrency control - when multiple students need to concurrently write to the same note, they are allowed to do it anywhere in the note at any time, consistency maintenance - replicas of the same notes on each member's GroupNotes applications are kept consistent throughout the session in the sense that each update to the notes is consistently applied to all replicas, the order in which updates are applied to the note is consistently maintained across all replicas, and all replicas are identical at the end of the session.

Polling management supports vertical interaction by offering the standard question-and-response feature provided by a clicker solution, e.g., the lecturer can post a quiz/question through the GroupNotes client and students can use their GroupNotes application to vote/answer, and nonstandard features, e.g., a student can use their GroupNotes application to interact with the lecturer (asking questions, giving feedback).

Offline management consists of services not used by the GroupNotes application or client during a lecture. In particular, user management is for a student to create their login accounts to the system, update their profiles (e.g., enrolled topics, groups they belong to), customise their preferences (e.g., preferred colour for their identities in a session, preferred role in a session), and so on.

Post-processing management is to reconcile lecture slides and notes (e.g., reconciling notes from each group, selected groups, or the entire class) to generate combined learning objects for students to revise the topic later or for the lecturer to have a better understanding on how well each slide of the lecture was delivered to students or monitor the performance of individual group/student.

Repository management is for students or the lecturer to view or download lecture slides, notes, or reconciled slides and notes they have access to.

\section{APPLICATION USER INTERFACE}

The smartphone app user interface design is based on the results of a needs finding questionnaire given to third year computer science topic students at our university[13]. Working scenarios and screenshots are provided to illustrate particular design choices, with reference back to the educational requirements.

Today's smartphone primarily uses a soft keyboard on a viewing screen ranging from approximately 3 to a maximum of 5 inches and our design focus will be on devices meeting these criteria. The working scenarios provided show the application's user interface from the point of view of a single student's device.

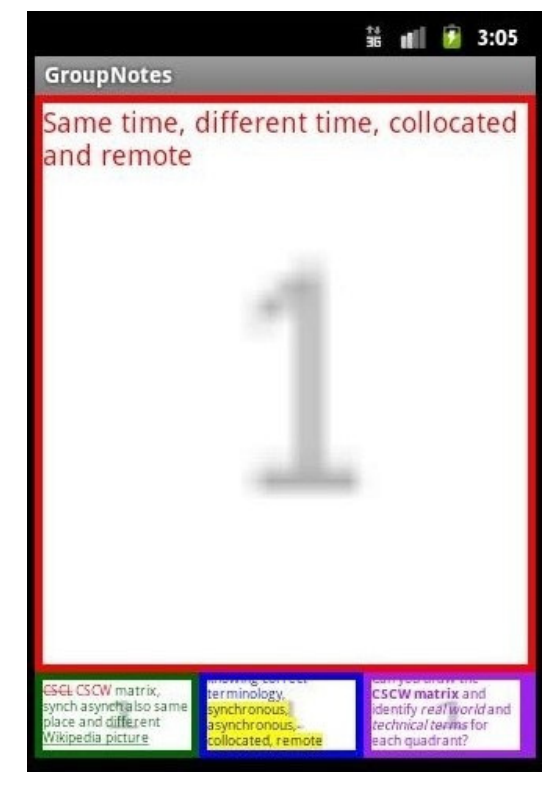

Fig. 8: One viewable editor and three radar views 
Fig. 8 shows the default screen view of the student after they have finished making a note. The screen is divided into the editor area (upper part) and the radar view area (lower part). The device owner can view and make notes in the editor area, while editors from their group members who have joined the session are shown in the radar view area. In this figure, Student 1's editor is shown in the editor area on the screen, while the editors from Students 2, 3 and 4, from left to right at the bottom of the screen, are shown in the radar view area. The background number in each editor, e.g., 1 in Student 1's editor indicates that the student is making a note for the first lecture slide.

In order for the student to view more editors, they need to drag editors from the radar view area to the editor area. In this example, there are four individual editors available representing the four student group members. The group may be made up of more or less students; four was identified from the questionnaire as being the maximum number of concurrent users an individual could keep up with cognitively during a lecture (to be tested). The available screen real estate limits the viewable editors possible.

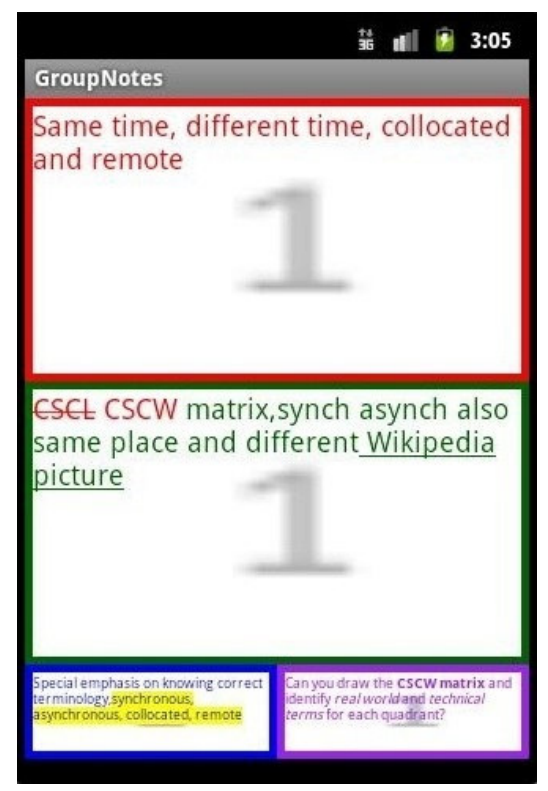

Fig. 9: Two viewable editors and two radar views

Figure 9 shows that the student has chosen to view two editors at the same time. This occurs by dragging Student 2's editor radar view (the bottom left editor in Figure 8) up to the editor area. To return to a single editor, which may or may not be the device owner's editor, would require the reverse action, i.e. dragging the unwanted editor down to the radar view area.

Figure 10 shows all four editors that take up all available screen real estate. It is worth pointing out that while a student can view up to four editors at the same time, when they start doing text entry in an editor, that editor will occupy the entire editor area, pushing the rest of editors from the editor area to the radar view area, primarily due to the significant screen real estate taken by the soft keyboard.

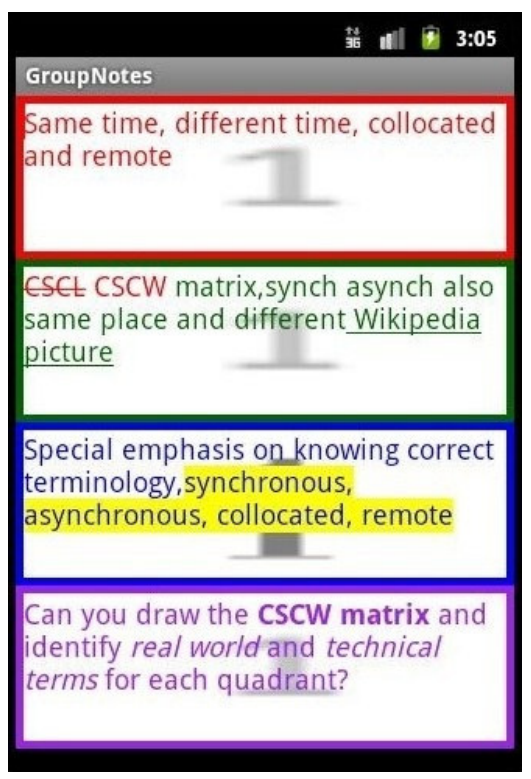

Fig. 10: All four viewable editors

\subsection{Text Editing Functions}

At present the text editor only has basic functions including the ability to change the colour of the text (system enforces the text colour and users are not allowed to), present text in plain, bold or italic, highlight the background of selected text, strikethrough selected text or underline selected text. These features will be accessed through a floating toolbar which will appear whenever text is selected. Figure 10 illustrates all of these features. Student 1 is using plain text in red in their own editor.

Student 2 has written notes in green in their editor to which Student 1 has performed a strikethrough of the acronym CSCL and then entered the correct acronym CSCW; both changes are in a different colour to identify an input from other than the owner of the editor. Student 2 has also highlighted something they consider important in their notes and has underlined the words Wikipedia picture.

Student 3 is using blue text and has provided emphasis on four words from their notes through highlighting.

Student 4 using the colour purple has used bold on the words CSCW matrix and italicised real world and technical terms for emphasis. 


\subsection{User Identification}

The questionnaire strongly suggested that users should be differentiated on the individual's device through the use of a unique colour for each group member. This colour borders the editor where that user writes their notes and is illustrated in each of Figures 8-10. The colour also identifies specific user generated content which is entered into another group members writing area, such as Figure 10 where Student 1 (in red) performed a strikethrough on the text CSCL written by the owner of editor 2 (in green).

CSCW is the new text written in this green area, also in red. This allows other group members to determine who has made the change.

\subsection{Note Synchronisation}

In a real-time collaborative note-taking session consisting of up to 4 students, each student is allocated a dedicated multi-page note that corresponds to the number of lecture slides. Each note can be jointly edited by the 4 students; therefore, the note-taking session is actually composed of 4 parallel collaborative editing sessions, one for each note.

We devised an optimistic concurrency control solution to synchronise each note by leveraging the GroupNotes Server as the mediator. In this solution, each note is replicated in every student's App and operations performed in one App are broadcast to all other Apps.

\section{CONCLUSIONS AND FUTURE WORK}

Our approach to increasing the engagement of students in lectures using smartphones is innovative, both in terms of the purpose that the device is being used for, but also the technical contributions allowing for the different learning abilities and styles. A single editor, where notes from all group members are intermingled and there is no opportunity for an individual to look at just a single group member's notes, does not meet the primary requirement for flexible and effective collaborative learning in lectures.

Design for multiple editors with each being explicitly owned by a student but allowing for collaborative editing of the same note provided the driving force behind the technical innovations outlined in this paper. The innovative collaborative interface allows any group member to freely write into any editor at any time, the optimistic note synchronisation solution allows a note to be fully synchronised without requiring much network bandwidth or any constraint on users' activities, and the novel operation buffer compression algorithm allows the
App to take full advantage of the available wireless networking resources and a smartphone's battery life.

Future work includes usability study of the GroupNotes system, including evaluation of the user interface and collaboration features as well as study of human factors. Studies into collaborative learning using the system will be carried out to determine the learning outcomes of students participating in the system compared to either nonparticipation or participation using alternative technologies.

\section{REFERENCES}

1. Bligh, D.A., What's The Use of Lectures? 2000, San Francisco, CA: Jossey-Bass.

2. Litchfield, A., et al. Using Students' Devices and a No-to-Low Cost Online Tool to Support Interactive Experiential mLearning. in Ninth IEEE International Conference on Advanced Learning Technologies. 2009. Riga, Latvia.

3. IDC. Australian Quarterly Mobile Device 2010-2014 Forecast and Analysis (1Q10). 2010 [cited 2010 November 4]; Available from: http://www.idc.com/research/.

4. Kam, M., et al., Livenotes: a system for cooperative and augmented note-taking in lectures, in Proceedings of the SIGCHI conference on Human factors in computing systems. 2005, ACM: Portland, Oregon, USA. p. 531 - 540.

5. Simon, B., et al., Noteblogging: taking note taking public, in Proceedings of the 39th SIGCSE technical symposium on Computer science education. 2008, ACM: Portland, OR, USA. p. 417421.

6. Hitchens, M. and R. Lister. A Focus Group Study of Student Attitudes to Lectures. in Eleventh Australasian Computing Education Conference (ACE 2009). 2009. Wellington, New Zealand: ACS.

7. Biggs, J.B., Teaching for Quality Learning at University. 1999, Buckingham: Open University Press.

8. Costa, J.C.E., T. Ojala., and J. Korhonen. Mobile Lecture Interaction: Making Technology and Learning Click. in IADIS International Conference Mobile Learning. 2008. Algarve, Portugal.

9. Falkner, K. and D.S. Munro. Easing the Transition: A Collaborative Learning Approach. in Eleventh Australasian Computing Education 
Conference (ACE 2009). 2009. Wellington, New Zealand: ACS.

10. Swype. Text Input for Screens. 2010 [cited 2010 Aug 10]; Available from: http://www.swypeinc.com/.

11. Swiftkey. Revolutionary text prediction for smartphones. 2010 [cited 2010 Aug 10]; Available from: http://www.swiftkey.net/.

12. Nuance. T9 Trace. 2011 [cited 2011 January 5]; Available from: http://www.nuance.com/for-business/by-product/t9trace/index.htm.

13. Reilly, M.D. and H. Shen. GroupNotes: encouraging proactive student engagement in lectures through collaborative note-taking on smartphones. in 9th International Conference on Computer Supported Collaborative Learning. CSCL2011: Connecting computer supported collaborative learning to policy and practice . 2011. Hong Kong.

14. Matheson, C., The educational value and effectiveness of lectures. The Clinical Teacher, 2008. 5(4): p. 218-221.

15. Perry, B., How the Brain Learns Best. Instructor; Primary ed, 2000. 110(4): p. 34.

16. Davis, R.C., et al., NotePals: lightweight note sharing by the group, for the group, in Proceedings of the SIGCHI conference on Human factors in computing systems: the $\mathrm{CHI}$ is the limit. 1999, ACM: Pittsburgh, Pennsylvania, United States. p. $338-345$

17. Patry, M., Clickers in Large Classes: From Student Perceptions Towards an Understanding of Best Practices. International Journal for the Scholarship of Teaching and Learning, 2009. 3(2).

18. Chickering, A.W. and Z.F. Gamson, Seven principles for good practice in undergraduate education. New directions for teaching and learning, 1991. 1991(47): p. 63-69.

19. Kirschner, F., F. Paas, and P.A. Kirschner, A Cognitive Load Approach to Collaborative Learning: United Brains for Complex Tasks. Educational Psychology Review, 2009(21): p. 12.

20. Anderson, L.W. and D.R. Krathwohl, $A$ taxonomy for learning, teaching, and assessing: $A$ revision of Bloom's taxonomy of educational objectives. 2001, New York: Longman.

21. Saville-Smith, $C$. and $P$. Kent, The use of palmtop computers for learning, A review of the
Literature. 2003, Learning and Skills Development Agency: London. p. Review of Literature.

22. Milrad, M., D. Bergman, and M. Jackson, $\mathrm{H}$. Exploring the Potential of Mobile Services to Support Learning and Communication in University Classes. in IEEE International Workshop on Wireless and Mobile Technologies in Education (WMTE'05). 2005. Tokushima, Japan

23. Lindquist, D., et al., Exploring the potential of mobile phones for active learning in the classroom, in Proceedings of the 38th SIGCSE technical symposium on Computer science education. 2007, ACM: Covington, Kentucky, USA. p. 384 - 388.

24. Aagard, H.P., D. Bowen. Kyle, and A. Olesova. Larisa (2010) Hotseat: Opening the Backchannel in Large Lectures. EDUCAUSE Quarterly 33.

25. Jarvela, S., Structuring and Regulating Collaborative Learning in Higher Education with Wireless Networks and Mobile Tools. Journal of educational technology \& society, 2007. 10(4): p. 71-79.

26. Ward, N. and H. Tatsukawa, A tool for taking class notes. International Journal of HumanComputer Studies, 2003. 59(6): p. 959-981.

27. EDUCAUSE. 7 Things You Should Know About Collaborative Editing. 7 Things You Should Know About... 2005 December 15; Available from: http://www.educause.edu/ELI/7ThingsYouShouldK nowAboutColla/156812.

28. Surowiecki, J., The Wisdom of Crowds. 2005, New York: Anchor. 